\title{
A Technique for Measuring the Equivalent RMS Input Noise of A/D Converters
}

\author{
T. MICHAEL SOUDERS, MEMBER, IEEE, AND JAMES A. LECHNER
}

\begin{abstract}
A simple accurate technique is described for measuring the equivalent rms input noise of $A / D$ converters. Noise can typically be measured with 10-percent accuracy in $1 \mathrm{~s}$, and the method has successfully been applied to converters with up to 16 bits of resolution. The measurements are made at input voltages corresponding to the test converter's decision levels, where the effects of noise are most pronounced. A feedback loop incorporating the unit under test locates and locks onto these levels. The method utilizes a theoretical relationship between the input noise and an expected number of counts derived digitally from the feedback loop response. A low-noise wideband operational amplifier is the only critical component required.
\end{abstract}

\section{INTRODUCTION}

$\mathrm{T}$ HE speed and accuracy limits of high performance A/D converters are frequently determined by inherent noise. Thus excess noise can often render higher conversion rates or higher resolution useless. Consequently, in the selection of converters for higher performance data acquisition systems, careful attention should be paid to their noise properties. A new technique is described here which makes possible quantitative objective noise measurements of A/D converters without requiring standards or other complex expensive equipment.

Unfortunately, several factors complicate the measurement of A/D converter noise. The noise cannot be measured directly as with many instruments but must be inferred from the response. Furthermore, the relationship between noise and response is complicated by the quantization process, because the effects of noise are more pronounced for input values near the decision levels represented by code transitions. Finally, these transition levels are not uniformly spaced in a real converter but are subject to local (differential) nonlinearity errors, which, in some measurements, are misinterpreted as noise. As treated in this work, the term "noise" is independent of this so-called "quantization noise," which produces systematic rather than random errors, and can in principle be reduced to negligible proportion by choosing a higher resolution converter.

A number of noise measurement techniques have been applied to A/D converters:

1) For the converter manufacturer, direct noise measurement data on the component parts, e.g., voltage reference, internal D/A converter network, analog comparator, and buffer amplifier, can be combined to estimate the overall equivalent input noise [1], [2]. This method

Manuscript received June 28, 1980.

T. M. Souders is with the Electrosystems Division, National Bureau of Standards, Washington, DC 20234.

J. A. Lechner is with the Statistical Engineering Division, National Bureau of Standards, Washington, DC 20234.

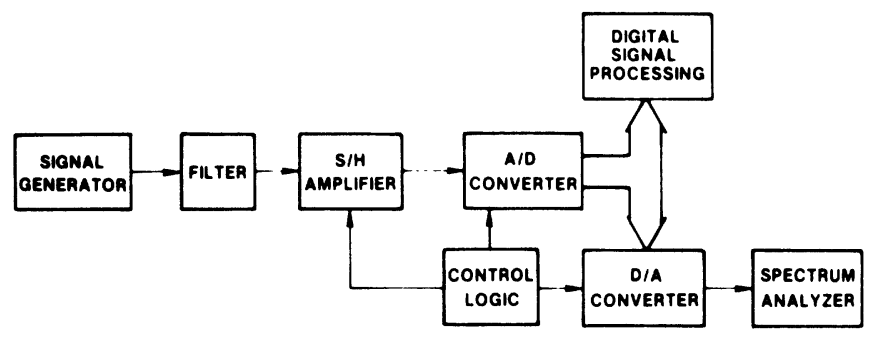

Fig. 1. Test method using spectrum analysis for A/D converter noise measurements.

produces only a rough estimate, and is generally unsuitable once the device has been assembled and packaged.

2) Spectrum analysis can be employed to measure the signal-to-noise ratio of dynamically exercised converters, as outlined in Fig. 1 [3], [4]. A pure sinewave, for instance, is digitized by the unit under test, and the data set is analyzed (in either the analog or digital domain) to measure distortion and noise contributed by the A/D converter. This technique is suitable for high-speed lower resolution converters used in communications, radar, and video systems, although it has several limitations. It is not capable of distinguishing among true converter noise, distortion caused by other converter errors such as nonlinearity, "quantization noise," and noise effects contributed by the sample/hold (S/H) amplifier. Such a separation of effects can be important, since an A/D converter need not always be used with a S/H amplifier (as, for instance, in the encoding of quasistatic multiplexed signals), and linearity errors can often be corrected by simple adjustment while the effects of inherent noise cannot, without sacrificing measurement speed. Furthermore, it becomes increasingly difficult to generate an adequately pure input signal for higher resolution (12-18 bits) converters.

3) Many of these limitations are overcome by providing the converter input from a higher resolution $D / A$ converter under computer control. Specific A/D transition levels can be located by incrementing the input voltage until the output codes fluctuate with equal probability about the transition. Having located the transition level, the input voltage is then offset by small known amounts and the probability distribution of output codes is again measured. A few such measurements are sufficient to determine the equivalent rms input noise, assuming a normal distribution. This technique is principally limited 


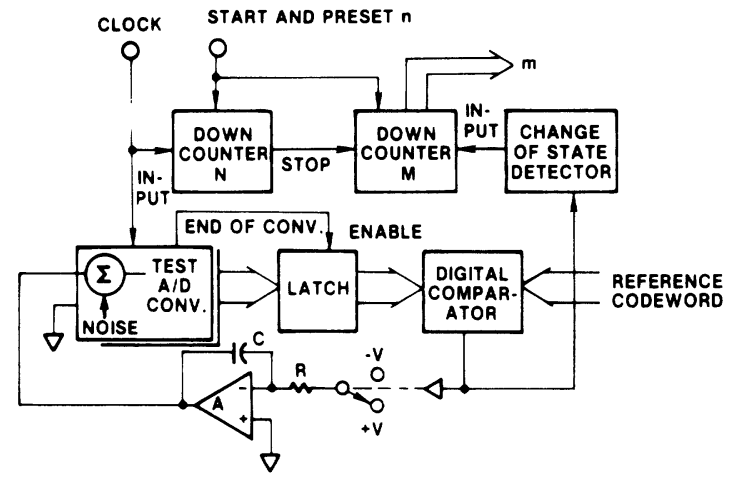

Fig. 2. New noise measurement technique utilizing feedback loop to lock onto transition levels, and digital circuitry to estimate probability of slope reversals.

by the availability of the required $\mathrm{D} / \mathrm{A}$ converter standard, which must have at least 3 bits $(\times 8)$ greater resolution than the converter under test, and a noise level commensurate with its resolution.

4) The dynamic crossplot method [5] can also provide noise information. Small regions of the converter transfer characteristic are graphically displayed on an oscilloscope which monitors the converter's response to a small-amplitude low-frequency (dither) signal superimposed on a dc level. While the technique is simple and convenient, and requires no expensive special-purpose equipment, the interpretation of the display is subjective, and the noise is therefore difficult to quantify accurately.

The noise measurement technique described in the following sections overcomes many of the limitations of these existing methods. Implementation can range from a simple manually operated set to a fully automatic measurement system. The only critical component required is a wide-band low-noise operational amplifier.

\section{APPROACH}

In the new technique, the noise measurements are made in the vicinity of the converter's decision levels represented by code transitions, where the noise sensitivity is greatest. These transition levels are located and locked on with a feedback loop controlling the input voltage to the converter under test (see Fig. 2). The feedback circuit has previously been applied by one of the authors, as well as others, in measuring other converter transfer characteristics, notably, linearity errors [6]. The actual rms noise measurement is based on a theoretical relationship between input noise and an expected number of counts derived from the response of the feedback circuit.

In operation, a digital comparator is used to compare the converter's digital output with the upper digital code of a selected transition. The input voltage to the converter is ramped up or down depending on whether or not the converter's output is less than the selected digital code. This is accomplished by having the comparator set the polarity of a reference input voltage $(V)$ to an operational integrator, and using the integrator output to feed the converter under test. Thus the input voltage to the converter will ramp up (or down) until the

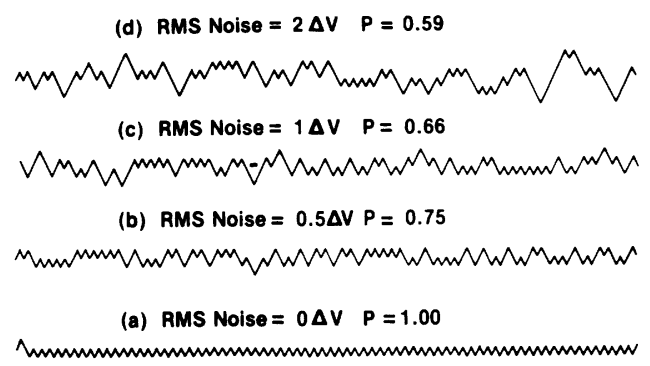

Fig. 3. Noise response of feedback loop: converter input voltage versus clock periods.

transition in question is detected, whereupon the slope of the integrator's output voltage reverses. The process continues at a preset clock rate maintaining the converter's input voltage very near the transition level. The precision with which the feedback loops locks on the transition is determined by the input voltage change per clock period $\Delta V= \pm V \Delta t / R C$, where $\pm V$ is the integrator input voltage, $R C$ is the integration time constant, and $\Delta t$ is the time between conversions.

With a noiseless A/D converter in the feedback loop, the input voltage change, once locking has occurred, will reverse its slope after each conversion, describing a triangular waveform with $\Delta V$ peak-to-peak amplitude, as in Fig. 3(a). The addition of random noise of rms value $\sigma$ at the A/D input causes a corresponding change in the feedback response, as shown in Fig. 3(b)-(d). The input voltage now follows a "random walk" about the transition level, reversing its slope with decreasing frequency (and straying farther from the transition level) as the noise level increases. If, at the given sampling rate, the successive values of noise are uncorrelated and follow a Gaussian distribution, the statistics of the random walk can be calculated in terms of the rms noise level.

An easily measured statistical parameter has been selected which can form the basis for an equivalent noise measurement made in terms of the voltage $\Delta V$. In particular, the probability $p$ of occurrence of a slope reversal following a conversion is easily measured with digital circuitry and can be related to noise level as described next. Since $\Delta V$ provides the reference voltage for the measurement of noise value $\sigma$, it is convenient to express $p$ in terms of the ratio $\sigma / \Delta V$.

It can be shown that the successive values of the sampled input voltage describe a discrete Markov chain for which, after each conversion, two possible moves exist, up by $\Delta V$ or down by $\Delta V$. The probability associated with each move is determined by both the displacement from the A/D transition level and the probability that noise of the opposite polarity will exceed this magnitude. For each level (quantized in increments of $\Delta V$ ) above and below the transition level, the probabilities can be calculated for moves to the next higher and lower states during the next clock period, based on the assumed noise properties. The move probabilities form a matrix from which the frequency with which each level is occupied can be determined. The probability of a slope reversal occurring is then the summation over all levels of the products of the probability of occupying each level and the probability of returning to that level in two moves. (For details, see the Appendix.) Note that for levels farther than a few $\sigma$ from the transition level, the 


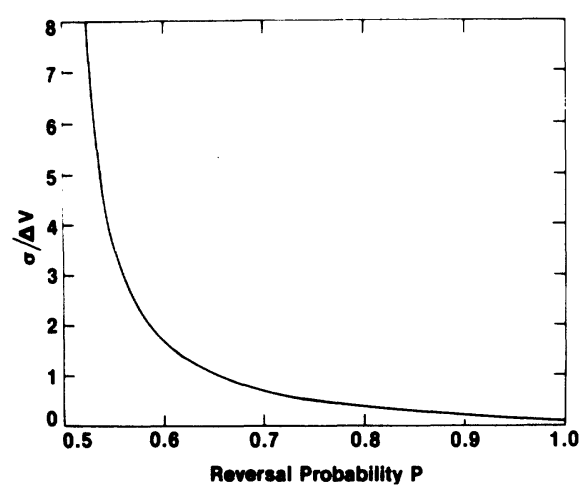

Fig. 4. Graph of relationship between the noise ratio $\sigma / \Delta V$ and the slope reversal probability $p$.

occupancy probabilities approach zero, so that only a relatively small number of levels need be considered to approximate the relation between slope reversal probability and the noise level.

A plot of the ratio $\sigma / \Delta V$ versus the calculated reversal probability $p$ is given in Fig. 4. Over the $\sigma / \Delta V$ range of 0.4 to 8.0 , this curve is described by the following expression:

$$
\sigma / \Delta V=\frac{A+B p}{1-2 p} \text { where } A=-0.70 ; \text { and } B=0.60
$$

with an accuracy of 1 percent in $\sigma / \Delta V$. These results assume that

1) the magnitude $\Delta V$ is constant for positive and negative rates of change,

2) noise from the integrating amplifier is negligible,

3) the noise has a Gaussian distribution, and at the given sampling rate, successive values are uncorrelated.

\section{IMPLEMENTATION}

The measurement of slope reversal probability can be accomplished with the digital circuitry functionally depicted in Fig. 2. The start command presets each down counter with the value $n$ and initiates counting. Changes in state of the digital comparator output (controlling the input voltage slope) decrement counter $M$ via the change-of-state detector until $n$ clock periods have been registered by counter $N$, stopping the test. The final value $m$ held in counter $M$, subtracted from and divided by $n$, is an estimate of the reversal probability. Thus, for example, for $n=100, n-m$ is the reversal probability in percent.

Several implementations of this basic technique are possible. For a simple manual test set offering pass-fail output capability, a toggle register can be used to select the reference codeword, and the counter $M$ can be preset with a reference value $m^{\prime}$, corresponding to $\sigma / \Delta V=1$, instead of $n$. An underflow condition on counter $M$ will then indicate the pass condition $\sigma<\Delta V$, which may be displayed by a panel indicator light. If $\Delta V$ is a calibrated variable, a wide range of $\sigma$ values can be measured. Alternatively, varying degrees of processing and control can be added to automatically set codewords, values of $\Delta V$, clock rate and sample size $n$, as well as to perform the noise calculations based on the data $m$. An automated implementation has been incorporated into a comprehensive test set for data converters described in reference [6].

\section{Measurement Precision}

The measurement time using this technique is determined by the clock rate and sample size $n$. While the former may be set to any value within the limitations imposed by the hardware and the unit under test, the latter is selected to give the desired precision.

The estimator of $p$ is $(n-m) / n$, and its standard deviation is at most $(p \cdot q / n)^{1 / 2}$, where $q=1-p$ (see Appendix). For any value of $p$, choosing $n=10^{4}$ gives a relative standard deviation (RSD) of at most 0.5 percent for the estimate of $p$. Because $|d \sigma / d p|$ is greater than unity, the uncertainty in $\sigma$ in greater: for $\sigma=1.0$, the RSD of the estimate of $\sigma$ is less than 4 percent. This RSD is approximately proportional to $\sigma$. Exact formulas are given in the Appendix. A suitable measurement of $\sigma$ with a $10-\mathrm{kHz}$ clock rate will take $1 \mathrm{~s}$.

\section{LIMITATIONS}

Potential error sources for this measurement technique fall into two categories: hardware limitations and deviations of the actual noise characteristics from the ideal model. Predominant errors of the first type include finite noise contributed by the integrating amplifier and mismatch error between the positive and negative rates of change of the voltage excursions $\Delta V$. The noise gain characteristic of an integrating amplifier reduces to a minimum the effects of amplifier noise (for which the noise gain is nearly unity) and attenuates input noise components of frequency $f$ to the integrator by the factor $1 / 2 \pi f R C$. These conditions are, therefore, generally more favorable than those for other amplifier configurations as, for example, might be used in a $\mathrm{D} / \mathrm{A}$ converter output. Mismatch errors in $\Delta V$ result primarily from offset voltage in the integrating amplifier, assuming some care has been taken to match input voltages $+V$ and $-V$. An offset voltage $V_{o s}$ causes a mismatch error of $2 V_{o s} / V$. Since the feedback loop maintains the ideal converter's input voltage within $\Delta V$ of the transition level, for a mismatch error $\epsilon$ one reversal will be lost for every $2 / \epsilon$ clock periods. It is offered, without proof, that the mismatch error, at worst, subtracts one half its value from the measured probability $p$. The equivalent effect on $\sigma$ can be found by applying the relationship between $\sigma / \Delta V$ and $p$, given earlier.

Deviations of the noise characteristics from the ideal model are a potentially more serious problem. Three types of deviation thought to be most likely to occur will be considered: hysteresis, alternating codes, and nonrandom noise. Both hysteresis and alternating codes can result from stray feedback mechanisms between digital and analog portions of the converter circuit and are most significant at major transitions. In the case of hysteresis, threshold levels defining a zone within which no code changes occur, are created on either side of the transition level. To produce an output code change, the input voltage must cross the zone, a condition which can render the noise algorithm useless if the size of the zone is significant with respect to $\Delta V$. Fortunately, a built-in test exists for this condition and has been verified through computer simulation. 
TABLE I

Verification Data, Presenting the Ratios $\sigma / \Delta V$ as MEASURED WITH AN RMS VOLTMETER, AND WITH THE A/D CONVERTER NOISE TESTSET

\begin{tabular}{|c|c|c|}
\hline \multicolumn{2}{|c|}{$\sigma / \Delta V$ as measured by } & Error \\
\hline (A) RMS Voltmeter & (B) Noise Test Set & $\frac{A-B}{A}$ \\
\hline 0.365 & 0.43 & -0.18 \\
\hline 0.500 & 0.55 & -0.10 \\
\hline 0.730 & 0.79 & -0.08 \\
\hline 1.00 & 1.08 & -0.08 \\
\hline 1.46 & 1.45 & +0.01 \\
\hline 2.00 & 1.90 & +0.05 \\
\hline 2.92 & 2.75 & +0.06 \\
\hline 5.84 & 5.39 & +0.08 \\
\hline
\end{tabular}

Regardless of $\sigma$, the measured probability $p$ will fall below 50 percent, the theoretical minimum value, when the interval between the thresholds exceeds $\Delta V$. Hysteresis can, therefore, be detected and measured by varying $\Delta V$ to find the point at which $p=0.5$.

Alternating codes create the opposite effect. In this case, the threshold levels define a zone within which the output codes reverse with 100 percent probability. For $\Delta V$ less than the threshold separation, the input voltage will remain within the zone, reversing each clock period unless noise is sufficient to exceed a threshold. Therefore, the noise will appear immeasurably small. Potential measurement errors due to either of these effects are best avoided by simply avoiding noise measurements at the major transitions.

Finally, lack of randomness of the sampled converter noise might occur, for example, if sufficient $1 / F$ noise predominates. The probability curve of Fig. 4 will no longer be valid. This condition is detected by performing the test at several values of $\Delta V$. A poor data fit indicates invalid conditions. Measurement results presented in the next section illustrate this test. If low-frequency noise components are responsible, a lower clock rate might provide a simple solution.

\section{VERIFICATION}

The validity of this test method has been established by applying well-characterized noise from an external noise generator to a second input of an A/D converter under test. The injected noise amplitude was set to at least five times the noise internal to the converter and was measured by a wide band true rms differential voltmeter. The ratios $\sigma / \Delta V$, as measured by voltmeter and noise test set, are presented in Table I for several values of $\Delta V$, along with the errors between

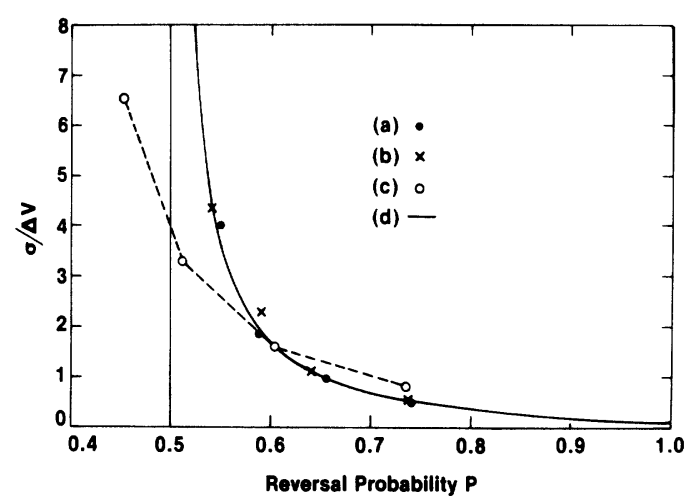

Fig. 5. Data illustrating effects of nonrandom noise. (a) Effective internal noise of typical test converter. (b) Measured response to externally generated Gaussian noise passed through a single-pole low-pass filter with a cutoff frequency equal to the sample rate. (c) Same as (b), cutoff frequency equal to $1 / 10$ sample rate. (d) Ideal noise curve.

the two measurements. With the exception of the lowest noise point $(\sigma=0.365 \Delta V)$, the two sets of measurements agree within 10 percent.

In contrast, the data plotted in Fig. 5 illustrate the effects of nonrandom noise on the measurement. Three sets of data are plotted corresponding to (a) the effective internal noise of a test converter, (b) the measured response to externally generated Gaussian noise passed through a single-pole low-pass filter with a cutoff frequency equal to the sample rate, and (c) the response to Gaussian noise filtered with a low-pass cutoff frequency of $1 / 10$ the sample rate. The ideal noise curve (d) is shown for reference. Note the strong deviation from ideal in example $c$ where the noise at successive clock cycles is highly correlated.

Measurements using this technique have been made on a number of $A / D$ converters ranging in resolution from 12 to 16 bits, with conversion times as low as $2 \mu$ s. Converters under test are commonly measured at a number of randomly chosen test codes to establish their average noise level. No measurement difficulties have arisen except at the major transitions where hysteresis has been encountered.

\section{APPENDIX}

This appendix presents details on the use of Markov chains in determining the relationship between the probability of a reversal and the noise level $\sigma$ and the precision of estimates of $\sigma$ obtained by this technique.

The sampled noise-free input voltage is confined to a set of equally spaced levels, with spacing denoted by $\Delta V$. By a linear transformation of this voltage, we can (without loss of generality) define a variable $y$ which takes on only integer values. These are such that $y=0$ corresponds to the first level below the A/D transition level and $y=1$ corresponds to the first level above the A/D transition level, i.e., the transition level is between $y=0$ and $y=1$. Let its value in this new scale be denoted by $d$. (See Fig. 6 for a diagram of the relationship between the input voltage $y$ and $d$.)

Now let $y(t)$ be the (transformed) input voltage at the $t$ th sample, for $t=1,2,3, \ldots$ In the absence of noise, $y(t)$ will alternate between 0 and 1 , so that every step will be a reversal. 


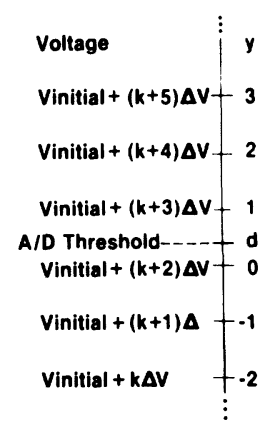

Fig. 6. Relationship between voltage, threshold level, and $y$.

On the other hand, when noise is present the value of $y$ will wander farther, in a probabilistic manner, as depicted in Fig. 3 . Let $N(t)$ be the instantaneous converter noise level, expressed in units of $\Delta V$, i.e., $N(t)$ is $(1 / \Delta V)$ times the actual noise level. Then, for given $y(t), y(t+1)$ will equal $y(t)+1$ if $N(t)$ is less than $d-y(t)$, because then $y(t)+N(t)$ will be less than the threshold $d$. Correspondingly, $y(t+1)$ will be $y(t)-1$ if $N(t)$ is greater than $d-y(t)$. Since it is assumed that successive values of $N(t)$ are uncorrelated and identically distributed, the probability of a step up (or down) depends on the current value of $y$ and not on $t$. Thus the behavior of $y(t)$ can be described by a set of "transition probabilities" $P_{i j}$, where $P_{i j}$ represents the probability of a transition from "state" $i$ to "state" $j$, i.e.,

$$
P_{i j}=\operatorname{Pr}(y(t+1)=j \mid y(t)=i) .
$$

In the particular case at hand, it is easily seen that $P_{i j}=0$ for $|i-j| \neq 1$, so that the structure of the so-called "transition matrix" $\boldsymbol{P}=\left(P_{i j}\right)$ is quite simple: all entries are zero except those bordering the main diagonal. (Theoretically, the dimension of $\boldsymbol{P}$ is infinite; but practically, any state which is far enough away from $d$ can be ignored, since the noise is unlikely to exceed several times its rms value.)

As $y(t)$ is defined here, it is in fact a (discrete-valued homogeneous) Markov chain. Such probabilistic processes have been studied extensively [7]. The next few paragraphs treat $y(t)$ from that point of view and derive results which can be used to calculate the probability of reversal as a function of the noise level.

This $y(t)$ is a discrete-valued function of discrete "time" $t(t=1,2, \ldots)$. The possible values of $y(t)$, which are called

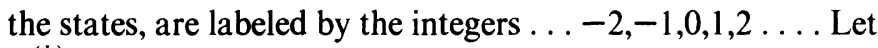
$P_{i j}^{(k)}$ represent the conditional probability that $y(t+k)=j$, given that $y(t)=i$, for any $t$. For $P_{i j}^{(1)}$ write simply $P_{i j}$. Let $P$ denote the matrix of $P_{i j}$. Furthermore, let $\theta=\left(\theta_{i}\right)$ denote a vector of so-called steady-state probabilities. The term steady-state means that if the state at time $t, y(t)$, is chosen at random according to the probabilities $\theta_{i}$, then the probability of being in any particular state $j$ at any later time is $\theta_{j}$. Mathematically, this implies that $\theta$ is a left eigenvector of the matrix $P$, with the corresponding eigenvalue equal to unity, i.e., $\theta$ satisfies the equation

$$
\theta^{\prime} P=\theta^{\prime}
$$

(By induction, then, $\theta^{\prime} P^{k}=\left(\theta^{\prime} P\right) P^{k-1}=\ldots=\theta^{\prime}$.)
Computer programs exist for finding such eigenvectors. However, in the case at hand, the solution can be found more simply by taking advantage of the fact that $P_{i j}=0$ unless $\mid i$ $-j \mid=1$. Equation (A2) becomes

$$
\theta_{i}=\theta_{i-1} P_{i-1, i}+\theta_{i+1} P_{i+1, i}, \quad \text { all } i \text {. }
$$

Let $b_{i}$ represent $P_{i, i+1}$. Then $1-b_{i}=P_{i, i-1}$, of course, and (A3) becomes

$$
\theta_{i}=\theta_{i-1} b_{i-1}+\theta_{i+1}\left(1-b_{i+1}\right), \quad \text { all } i .
$$

It can easily be verified (by substitution) that this relation is satisfied if the $\theta_{i}$ satisfy the simple recursion relationship

$$
\theta_{i}=\theta_{i-1} b_{i-1} /\left(1-b_{i}\right), \quad \text { all } i \text {. }
$$

Since it is physically necessary that the $\theta_{i}$ are large for $i$ near zero, and become very small as $i$ departs from zero, it is useful to express all the $\theta_{i}$ in terms of $\theta_{0}$. Let $r_{i}=\theta_{i} / \theta_{0}$ (so that $r_{0}=$ 1). Now (A5) becomes

$$
r_{i}=r_{i-1} b_{i-1} /\left(1-b_{i}\right), \quad r_{0}=1
$$

which allows the computation of all the $r_{i}$. Finally, since the $\theta_{i}$, being probabilities, must sum to unity, it follows that

$$
\sum_{i} r_{i}=\left(1 / \theta_{0}\right) \sum_{i} \theta_{i}=1 / \theta_{0}
$$

and each $\theta_{i}$ is obtained as

$$
\theta_{i}=r_{i}\left(\sum_{i} r_{i}\right)^{-1} \text {. }
$$

An expression for the probability of a reversal can be obtained as follows:

A reversal will occur at time $t$, if and only if $y(t-1)=y(t$ $+1)$. The probability of going from state $i$ to state $j$ in two steps is denoted by $P_{i j}^{(2)}$, and is the $i, j$ th entry in the matrix $P^{(2)}$ $=P \cdot P$. To see this, simply note that by definition

$$
P_{i j}^{(2)}=\sum_{k} P_{i k} P_{k j}
$$

is the sum over all $k$ of the probability of going from $i$ to $k$ and then from $k$ to $j$; there is no other route from $i$ to $j$ in two steps; and these routes are mutually exclusive, so that their probabilities can be added. Thus the probability of a reversal at $t$, $p(t)$, is given by

$$
p(t)=\sum_{i} P(y(t-1)=i) P_{i i}^{(2)}=\sum_{i} \theta_{i} P_{i i}^{(2)} .
$$

Both the $\theta_{i}$ and the $P_{i i}^{(2)}$ can be calculated once the $P_{i j}$ are specified. This will now be done.

Suppose the true converter noise to be Gaussian with mean zero and rms amplitude $\sigma$. Then $N(t)$ is Gaussian with mean zero and rms amplitude $\sigma / \Delta V$. It follows, then, that

$$
\begin{gathered}
P_{i, i+1}=\Phi\left(\frac{d-i}{\sigma / \Delta V}\right) \\
P_{i, i-1}=1-\Phi\left(\frac{d-i}{\sigma / \Delta V}\right)=\Phi\left(\frac{i-d}{\sigma / \Delta V}\right)
\end{gathered}
$$

where $\Phi(z)$ is the standard Gaussian cumulative distribution 
function

$$
\Phi(z)=(2 \pi) \frac{-1}{2} \int_{-\infty}^{z} \exp -\left(t^{2} / 2\right) d t .
$$

From (A8) and (A10)

$$
\begin{gathered}
P_{i i}^{(2)}=P_{i, i-1} P_{i-1, i}+P_{i, i+1} P_{i+1, i} \\
=\Phi\left(\frac{i-d}{\sigma / \Delta V}\right) \Phi\left(\frac{d-i+1}{\sigma / \Delta V}\right)+\Phi\left(\frac{d-i}{\sigma / \Delta V}\right) \Phi\left(\frac{i+1-d}{\sigma / \Delta V}\right) .
\end{gathered}
$$

Also, $b_{i}=\Phi((i-d) /(\sigma / \Delta V))$, so that (A6) and (A7) can be used to compute $\theta$. The computation begins effectively at $\theta_{0}$, and proceeds in both directions; it need not be carried out beyond $|i|=4 \sigma / \Delta V$, or $|i|=4$, whichever is larger, since the remaining $\theta_{i}$ are less than $10^{-7}$.

By definition, the range of $d$ is $(0,1)$. However, since $N(t)$ has a symmetric distribution, the probability of reversal for $d=c>1 / 2$ is the same as for $d=1-c$. Therefore, values of $d$ were restricted to the range $(0,1 / 2)$. The calculations show that $p$ is nearly insensitive to $d$, unless $\sigma / \Delta V$ is less than 1 . Since the offset between the initial input voltage and the chosen threshold can be many times the step size $\Delta V$, the actual values of $d$ on repeated trials are likely to appear to be randomly drawn from a uniform distribution over the interval $(0,1)$. Thus the values of $p$ for $\sigma / \Delta V<1$ were obtained by calculating $p$ for $d=0,0.1,0.2,0.3,0.4$, and 0.5 , and then averaging these with weights $1,2,2,2,2,1$, respectively. Even for $\sigma / \Delta V$ as small as $1 / 2, p$ is a smooth, almost linear function of $d$, varying from about 0.73 at $d=0$ to 0.77 at $d=1 / 2$. For lower values of $\sigma / \Delta V$, the linearity ceases to hold: for $\sigma / \Delta V=0.1$, for example, $p=0.75$ for $d=0,0.994$ for $d=0.25$, and 1.0 for $d=$ 0.5 .

The relationship between $p$ and $\sigma / \Delta V$, as shown in Fig. 4 , is used to convert estimates of $p$ into estimates of $\sigma$. In order to facilitate this conversion, a rational function was fitted to the calculated points. The simplest such function which fits the data well is

$$
\sigma / \Delta V=\frac{-0.7+0.6 p}{1-2 p}
$$

The slope of this curve is

$$
\frac{d(\sigma / \Delta V)}{d p}=\frac{-0.8}{(1-2 p)^{2}} .
$$

The relative standard deviation (RSD) of an estimate $\hat{\sigma} / \Delta V$ of the true value $\sigma / \Delta V$ is defined to be $\operatorname{SD}(\hat{\sigma} / \Delta V) / \sigma / \Delta V$. It is given by

$$
\operatorname{RSD}(\hat{\sigma} / \Delta V)=(\sigma / \Delta V)^{-1}\left|\frac{d}{d p}\left(\begin{array}{c}
\sigma \\
\Delta V
\end{array}\right)\right| \operatorname{var}(\bar{p})^{1 / 2}
$$

where $\bar{p}$ is the corresponding estimate of $p$. Now, if the probability of a reversal were the same for each conversion and the conversions were independent trials, then the variance of $\bar{p}$ would be the well-known formula $(p q / n)$. In the case at hand, however, the value of $y(t)$ wanders about the threshold level, and the probability of a reversal at $t$ depends on the value $y(t$

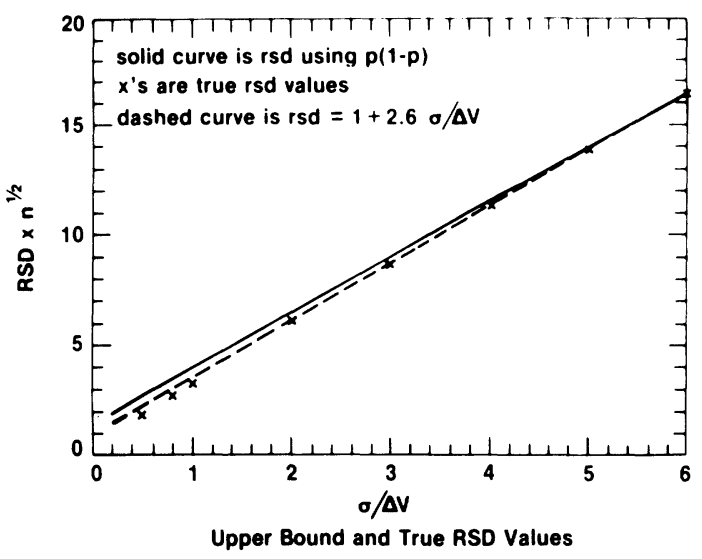

Fig. 7. Relative standard deviation of the estimate of $\sigma / \Delta V$, as a function of $\sigma / \Delta V$.

$-1)$. The technique measures an average $p$ : in fact, the value measured is an estimate of

$$
p^{*}=\sum_{i} \theta_{i} P_{l i}^{(2)}
$$

The variance of such an estimate is [8]

$$
\operatorname{var}(\bar{p})=n^{-1} \sum_{i} \theta_{i} P_{i i}^{(2)}\left(1-P_{i i}^{(2)}\right)
$$

which is bounded above by the simpler expression $n^{-1} p^{*}(1-$ $\left.p^{*}\right)$. In fact, for the case under consideration, $\operatorname{var}(\bar{p})$ approaches $n^{-1}\left(p^{*}\left(1-p^{*}\right)\right.$ as $\sigma / \Delta V$ increases; it is 19 percent less for $\sigma / \Delta V=1,6$ percent less for $\sigma / \Delta V=2$, and only $1 \frac{1}{2}$ percent less for $\sigma / \Delta V=4$.

Since the true variances are available from (A15), they were used with (A13) and (A12) to determine a few RSD values for $\hat{\sigma} / \Delta V$. These values are shown on Fig. 7 , along with the curve

$$
\operatorname{RSD}(\hat{\sigma} / \Delta V)=n^{-1 / 2}(1+2.6 \sigma / \Delta V)
$$

which is a good upper bound for the RSD values, and the curve obtained by using $\sigma^{2}(\bar{p})=p q / n$ in (A13).

Note that by changing $\Delta V$, a suitable RSD of $\hat{\sigma}$ can always be found, provided a suitable value of $n$ has been chosen, for example, $n=10^{4}$.

\section{REFERENCES}

[1] B. M. Gordon, "Noise-effects on analog to digital conversion accuracypart 1," Comput. Des., Mar. 1974.

[2] - -, "Noise-effects on analog to digital conversion accuracy-part 2," Comput. Des., Apr. 1974.

[3] W. A. Kester, "Characterizing and testing A/D and D/A converters for color video applications, IEEE Trans. Circuits Syst., vol. CAS-25, July 1975.

[4] B. Pratt, "Test A/D converters digitally," Electron. Des. 25, Dec. 6, 1975

[5] D. Sheingold, Ed., Analog-Digital Conversion Handbook. Norwood, MA: Analog Devices, 1972.

[6] T. M. Souders and D. R. Flach, "An automated test set for high resolution analog-to-digital and digital-to-analog converters," IEEE Trans. Instrum. Meas., vol. IM-28, Dec. 1979.

[7] D. R. Cox and H. D. Miller, The Theory of Stochastic Processes. New York: Wiley, 1965

[8] W. Hoeffding, "On the distribution of the number of successes in independent trials," Ann. Mathemat. Stat., vol. 27, 1956. 